\section{What influences treatment satisfaction in patients with personality disorders? A naturalistic investigation in a hospitalization setting}

\author{
Stefan Gebhardt, ${ }^{1}$ Martin Tobias Huber ${ }^{1,2}$ \\ 1Department of Psychiatry and \\ Psychotherapy, University of Marburg; \\ 2Department of Psychiatry, \\ Psychotherapy and Psychosomatics, \\ Hospital of Stade, Germany
}

\section{Abstract}

Treatment satisfaction of different mental disorders is still poorly understood, but of high clinical interest. Inpatients of a general psychiatric care hospital were asked to fill out questionnaires on satisfaction and clinical variables at admission and discharge. On the basis of an exploratory approach, differences in treatment satisfaction among diagnostic groups were examined by means of one-way analysis of variance. Potential associated clinical and socio-demographic variables were studied using multi/univariate tests. Patients with personality disorders $(n=18)$ showed a significantly lower treatment satisfaction (ZUF-8, Zurich Satisfaction Questionnaire) and a slightly lower improvement of symptoms (CGI, Clinical Global Impression) and global functioning (GAF, Global Assessment of Functioning scale) than that of other diagnostic groups $(n=95)$. Satisfaction in patients with personality disorders correlated much stronger with the symptom improvement and slightly with the functioning level than in patients without personality disorders. Interestingly, in patients with personality disorders psychopharmacological treatment in general (present versus not present) was independent from satisfaction. This exploratory investigation suggests that a lower satisfaction of patients with personality disorders in a general psychiatric hospital is mainly based on a reduced improvement of the symptoms and of the global functioning level.

\section{Introduction}

Patient satisfaction with treatment is more and more recognized as one of the worthiest parameters of treatment success and has a strong impact on institutional quality management. Departments of psychiatry are still in the beginning of developing standards to increase patient satisfaction. 1 Therefore, patient satisfaction as a subjective criterion of improvement of life quality has a crucial medical dimension as well as a strong role in clinic economics. However, influencing factors are still little understood.

According to the current state of research the following main results on symptomatology, psychopharmacology and diagnosis in relationship to patient satisfaction could be found.

Treatment satisfaction is significantly associated with symptom reduction and low symptom severity and a high global functioning at the end of the treatment.2,3 In outpatients it could be found that coping with specific problems and symptoms were associated with satisfaction among male patients, whereas changes in the interpersonal domain were important outcomes associated with patient satisfaction in female patients. ${ }^{2}$ In a study on inpatients at a psychiatric unit patient satisfaction correlated negatively with depression scores and personality pathology, whereas length of stay, age and sex contributed minimally. ${ }^{4}$ In another study patient satisfaction correlated negatively with severity of depressive symptoms at discharge and number of comorbidities in major depressive disorder. ${ }^{5}$

A meta-analysis suggested that patients with psychiatric disorders preferred a psychological treatment opposed to a psychopharmacological treatment, especially in case of younger patients and women. ${ }^{6}$ In an older study pharmacotherapy itself was not related to patient satisfaction, but patients who perceived improvements in pharmacotherapy as one of the most important treatment outcomes were less satisfied than others. ${ }^{2}$ Additionally, we could describe an association of a reduced treatment satisfaction with pharmacological problems. ${ }^{3}$ It could be shown that the number of prescribed drugs correlated negatively with patient satisfaction in both patients with depression and schizophrenia. ${ }^{5}$

Furthermore, the psychiatric diagnosis seems to be relevant with respect to patient satisfaction. In an outpatient study patients with somatoform, eating, and personality disorders were shown to be less satisfied than patients with affective, anxiety, and adjustment disorders. ${ }^{2}$ Other studies suggest a reduced satisfaction in patients with substance abuse compared to patients with a major depressive episode. ${ }^{7}$ In a post-hoc analysis of six studies of patients with major depressive disorder the change in satisfaction with antidepressant drugs from baseline to endpoint was significantly correlated with symptomatic improvement on the depressive symptoms,${ }^{8}$ a comparable result was found in another study. 5 Similarly, in patients with schizophrenia depression score is correlating negatively with the subjective well-being score. ${ }^{9} \mathrm{~A}$ recent study showed no differences between
Correspondence: Stefan Gebhardt, Department of Psychiatry and Psychotherapy, University of Marburg, Rudolf-Bultmann-Str. 8, D-35033 Marburg, Germany.

Tel.: +49.6421.58.65.200 - Fax: +49.6421.58.68.939.

E-mail: Stefan.Gebhardt@uni-marburg.de

Key words: Satisfaction; Personality disorders; Psychiatric inpatients; Psychopharmacology; Psychotherapy; Treatment.

Acknowledgements: the authors would thank the staff of the hospital for supporting this study, especially Mrs. Dr. Anna Maria Wolak, as well as the patients who participated in this study. We thank Mr. Prof. J.-C. Krieg (Marburg/Germany) and Mrs. Joanna Adamowicz (Hanau/Germany) for their helpful comments on the manuscript.

Contributions: SG, research design, asssembly of data, statistical analysis, data analysis and interpretation, writing the article, critical revision of the article, final approval of article; MTH, conception and design of the research project, collection and assembly of data, data analysis and interpretation, critical revision of the article, final approval of article.

Conflict of interest: the authors declare no conflict of interest.

Received for publication: 5 September 2016. Revision received: 1 November 2016.

Accepted for publication: 2 November 2016.

This work is licensed under a Creative Commons Attribution-NonCommercial 4.0 International License (CC BY-NC 4.0).

(C) Copyright S. Gebhardt and M.T. Huber, 2016 Licensee PAGEPress, Italy

Mental Illness 2016; 8:6868

doi:10.4081/mi.2016.6868

patients with major depressive disorder and those with schizophrenia in patient satisfaction; however, patients with depressive disorder and comorbid personality disorder showed a lower satisfaction than depressive patients without a personality disorder. ${ }^{5}$ In a previous study it could be shown that global subjective quality of life was lower in patients with personality disorders compared to patients with major mental (psychotic) disorders. ${ }^{10}$

The variables age, sex and education seem to have no relevant influence on satisfaction, whereas race in case of minority groups, patients in mental health care, psychiatric diagnosis, chronicity of illness, poor prognosis, compulsorily detained patients obviously show lower satisfaction levels. ${ }^{11}$

However, the current state of research is still scarce. In the present investigation subsequent to our main hypothesis-testing approach, 3 we now used an exploratory 
approach to focus on these three dimensions (symptom severity, psychopharmacology, diagnosis) with respect to satisfaction and in relationship to each other. We followed this procedure: firstly, to explore differences in treatment satisfaction among specific diagnostic groups; secondly, to identify significant differences in treatment and socio-demographic variables of the patients groups who are differing in treatment satisfaction.

\section{Materials and Methods}

\section{Sample description}

113 inpatients (52.8\%; 59 females, 54 males; mean age at discharge $48.3 \pm 16.6$ years; range 18.5-87.3 years; mean inpatient treatment duration $1.4 \pm 1.2$ months) admitted to the Department of Psychiatry, Psychotherapy and Psychosomatics, Stade City Hospital, Germany, between May and August 2009 were investigated. The psychiatric hospital at the Elbe Klinikum Stade is an academic teaching hospital containing three general psychiatric inpatient wards for 80 patients, a day clinic with 23 places and an outpatient department. The hospital is responsible for the complete inpatient psychiatric care of the county of Stade with approximately 200.000 inhabitants. Per year about 1.300 inpatients are treated.

42 patients had been admitted by their general practitioner, 13 by their psychiatrist, 13 by the outpatient department of the clinic, 6 by an emergency doctor; 23 patients had requested an admission by themselves, 8 had been transferred from another ward, 3 from another clinic, 5 had been admitted due to other reasons. Of the 113 patients 4 patients were treated involuntarily. 7 patients had a private health insurance.

The patients were diagnosed according to the International Classification of Diseases ICD-10,12 using (semi-)structured diagnostic interviews with the diagnostic groups organic, including symptomatic, mental disorders (F0, $\mathrm{n}=6$ ), mental and behavioral disorders due to psychoactive substance use ( $\mathrm{F} 1, \mathrm{n}=26)$, schizophrenia, schizotypal and delusional disorders (F2; n=17), mood [affective] disorders (F3, $\mathrm{n}=48$ ), neurotic, stress-related and somatoform disorders $(\mathrm{F} 4, \mathrm{n}=12)$, behavioral syndromes associated with physiological disturbances and physical factors ( $\mathrm{F} 5, \mathrm{n}=0$ ), disorders of adult personality and behavior $(\mathrm{F} 6, \mathrm{n}=3$ as main diagnosis and $n=15$ as comorbid (often basic) diagnosis), mental retardation $(\mathrm{F} 7, \mathrm{n}=1) \cdot{ }^{3}$ Further data can be drawn from our first report.

Patients gave written informed consent; the study has been performed in accordance with the ethical standards laid down in the 1964
Declaration of Helsinki and its later amendments (approved at $3^{\text {rd }}$ November 2008 by the Ärztekammer Niedersachsen Hannover; State Medical Chamber of Lower Saxony, Germany).

The average duration since psychiatric diagnosis was $5.1 \pm 7.9$ years. Mean inpatient treatment duration was $1.4 \pm 1.2$ months. Thirtythree patients $(29.2 \%)$ received antidepressants, 25 (22.1\%) antipsychotics, and 34 $(30.1 \%)$ both in combination; 13 patients (11.5\%) received no medication. Psychotherapy was predominantly or concomitantly used in those mental disorders which profit from psychotherapy according to the current state of research, e.g. neurotic disorders or personality disorders. The psychotherapy was mainly based on cognitive behavioral therapy with psychodynamic elements.

\section{Instruments}

The ZUF-8 (Zurich Satisfaction Questionnaire; a questionnaire of patient satisfaction) ${ }^{13}$ was applied at discharge. It represents a reliable and valid self-evaluation questionnaire of patient satisfaction with the treatment. In our study sample reliability analysis revealed a good internal consistency (Cronbach's alpha 0.886). For the evaluation of general treatment variables as well as the psychosocial functioning the BADO (Basisdokumentation; basic documentation) ${ }^{14}$ questionnaire was used including self-rated CGI (Clinical Global Impression scale) ${ }^{15}$ and physician-rated GAF (Global Assessment of Functioning scale of the American Psychiatric Association in a German Version) ${ }^{16}$ at admission and discharge.

\section{Statistical procedures and variables}

Four main calculations were performed: First, differences in ZUF-8 total score between the diagnostic groups were examined using Oneway ANOVA analysis with post-hoc-tests (Scheffé). The diagnostic groups F5 and F7 were excluded from the analysis because the group contained no more than one patient.

In a second analysis a t-test on the ZUF-8 total score with independent variables on all patients diagnosed with a personality disorder including comorbid personality disorders $(\mathrm{n}=18)$ versus the remaining patients $(\mathrm{n}=95)$ was underdone.

In the third analysis the patient groups with $(n=18)$ and without $(n=95)$ personality disorders were compared with respect to the following treatment and socio-demographic variables: age, gender, school examination level, inpatient treatment duration, duration of the mental disorder, number of hospitalizations, duration of the current symptom manifestation, number of somatic diagnoses, psychopharmacological treatment (versus none), use of antidepressants, pharmacological problems (treatment resistance, considerable adverse events, lacking compliance of the patient), CGI at admission and at discharge (CGI part 1), CGI change (CGI part 2), GAF at admission and at discharge, GAF difference between admission and discharge, an addiction disorder as main or comorbid disorder, single items of the ZUF-8.

Differences between patient groups were studied using multi/univariate variance analyses (Pillai's trace) and Student's $t$-tests for continuous variables and chi-square test for dichotomous data. Furthermore, the MannWhitney-U-test was used in case of ordinal dependent variables or non-normally distributed variables identified by the KolmogorovSmirnov-test.

Finally, variables were identified which correlate with patient satisfaction (Pearson correlation) or are associated (in nominal variables, $t$-tests) within the group of personality disorders. A multivariate analysis was used to identify the main impact of these dependent variables on the differences between the two patient groups.

Additionally, to elucidate the improvement in both patient groups, a longitudinal analysis on CGI part 1 at admission to discharge was conducted for each group separately (Student's $t$-test for dependent samples).

All P-values were two-tailed; 0.05 was the significance level. A correction for multiple testing was not included because of the exploratory nature of our study. The data were analyzed using Statistical Package of the Social Sciences (SPSS 21.0 for Windows) software.

\section{Results}

In the first calculation significant differences in ZUF-8 total score between the diagnostic groups were detected $(\mathrm{P}<0.001)$. In the post-hoc-tests, personality disorders proved to be different from each other diagnostic group $(\mathrm{P}<0.001-\mathrm{P}=0.001)$, whereas no other diagnostic group showed differences among each other.

In the second analysis, the group of personality disorders displayed a significantly reduced patient satisfaction $(n=18$; mean score $25.7 \pm 6.3$ ) compared to the patients without personality disorders $(\mathrm{n}=95$; mean score $27.2 \pm 3.4)(\mathrm{P}=0.019, t$-test). The subgroup of personality disorders $(n=18)$ consisted of 8 patients suffering from a combined personality disorder, 3 from a dependent, 2 from an emotional unstable, 2 from a histrionic, 2 from another (narcissistic) personality disorder and 1 from an otherwise non-specified personality disorder.

The third analysis showed the following results (see also Table 1).

I) with respect to the change of parameters 
during the treatment (CGI part 2 and the GAF change): Patients with personality disorders showed a significantly lower improvement of symptoms during the treatment (CGI part 2: 3.5 vs. 3.1; Mann-WhitneyU-test: $\quad \mathrm{P}=0.019$; and $\mathrm{GAF}$ change: $+18.3 \pm 14.7$ vs. $+25.8 \pm 14.5$; Mann-WhitneyU-test: $\mathrm{P}=0.026$ ). Obviously, patients with personality disorders have been rated slightly worse in the improvement of psychiatric symptoms and general functioning during the psychiatric treatment compared to the patients without personality disorders.

II) With respect to the endpoint status at discharge (CGI part 1 and GAF): However, at the time of discharge, no significant difference in the symptom severity/level of global functioning could be revealed: the CGI part $1 /$ GAF score at discharge was $4.5 \pm 1.2 / 64.4 \pm 11.6$ in personality disorders and $4.2 \pm 1.2 / 67.0 \pm 11.6$ in other diagnoses (Mann-Whitney-U-tests). The longitudinal analysis within the patient group of personality disorders, or the non-personality group, respectively, also revealed a good symptom improvement (CGI part 1) in both the personality disorder group (CGI part 1 at admission $6.0 \pm 0.6$; at discharge $4.5 \pm 1.2 ; \mathrm{P}<0.001$ ) and the non-personality disorder group (CGI part 1 at admission $6.2 \pm 0.8$; at discharge $4.2 \pm 1.2 ; \quad \mathrm{P}<0.001$ ) (Student's $t$-test for dependent samples).
The examination of associated variables revealed the following results. Patients with personality disorders were significant younger than patients with no personality disorders $(40.1 \pm 13.2$ vs. $49.9 \pm 16.7$ years; $t$-test: $\mathrm{P}=0.022)$. Furthermore, in patients with personality disorders the duration of the current symptom manifestation was longer (in the mean higher than 6 months) (Mann-WhitneyU-test: $\mathrm{P}=0.011$ ) and the inpatient treatment duration slightly longer $(2.0 \pm 1.4$ vs. $1.3 \pm 1.2$ months; $t$-test: $\mathrm{P}=0.056$, trend) compared to the remaining patients. Further, patients with personality disorders showed a trend towards a lower number of somatic diagnoses $(0.7 \pm 0.8$ vs. $1.1 \pm 1.5$; $t$-test: $\mathrm{P}=0.070$ ) and a better occupational level as well as a better job situation (Mann-Whitney-U-Test; $\quad \mathrm{P}=0.007 / \mathrm{P}=0.085$ ) compared to patients without personality disorders. Finally, the use of antidepressants was slightly more frequent in patients with personality disorders (14 patients vs. 4) compared to the other patients (53 vs. 42) (Mann-WhitneyU-test; trend: $\mathrm{P}=0.083$ ).

Correlations with patient satisfaction of the variables which were associated with the diagnosis of a personality disorder were also evaluated in the group of other diagnoses. Hereby we found only a positive correlation of patient satisfaction with the job situation $(\mathrm{r}=0.233$; $\mathrm{P}=0.025)$ and - as a trend - with the symptom improvement (CGI part 2; $\mathrm{r}=-0.185 ; \mathrm{P}=0.072$ ), but not with other variables including GAF at discharge or delta GAF.

Patients with personality disorders and those without did not differ in the variables: gender, school examination level, duration of the mental disorder (anamnestic data), number of hospitalizations, general psychopharmacological treatment (versus none), pharmacological problems, CGI part 1 and GAF at admission, an addiction disorder as main or comorbid disorder ( $n=37$ ), single Zuf-8 items.

Among the patients with personality disorders, patient satisfaction correlated strongly negatively with a symptom worsening (CGI part $2 ; \mathrm{r}=-0.768 ; \mathrm{P}<0.001$ ), whereas in patients without personality disorders the correlation was much weaker and only a trend (CGI part 2; $r=-0.185 ; P=0.072$; Figure 1). Furthermore, among the patients with personality disorders, patient satisfaction correlated as a trend positively with the improvement of the functioning level (delta GAF; $\mathrm{r}=0.435 ; \mathrm{P}=0.071$ ), whereas no significant correlation could be revealed in patients without personality disorders. Additionally, the use of antidepressants was positively associated with an increased patient satisfaction as a trend $(\mathrm{P}=0.092 ; t$-test $)$ in patients with personality disorders, but not in those without the diagnoses of a personality disorder.

Finally, in the multivariate analysis on the total sample we checked the impact of these

Table 1. Mean values of the tested variables and their statistical differences between patients with personality disorders and those without.

\begin{tabular}{|c|c|c|c|}
\hline Variables & $\begin{array}{l}\text { Personality disorders } \\
\qquad(\mathrm{n}=8), \\
\text { mean value/SD }\end{array}$ & $\begin{array}{l}\text { Other diagnoses } \\
\qquad(\mathrm{n}=95), \\
\text { mean value/SD }\end{array}$ & $\begin{array}{l}\text { Statistical } \\
\text { differences, } \\
\text { P-value }\end{array}$ \\
\hline Age (years) & $40.1 \pm 13.2$ & $49.9 \pm 16.7$ & $0.022^{*}$ \\
\hline Gender $(\mathrm{m} / \mathrm{f})$ & $6 / 12$ & $48 / 47$ & n.s. ${ }^{\circ}$ \\
\hline ZUF-8 sum score & $25.7 \pm 6.3$ & $27.2 \pm 3.4$ & $0.019 *$ \\
\hline CGI part 1 at admission & $6.0 \pm 0.6$ & $6.2 \pm 0.8$ & n.s.* \\
\hline CGI part 1 at discharge & $4.5 \pm 1.2$ & $4.2 \pm 1.2$ & n.s.\# \\
\hline CGI part 2 at discharge & $3.5 \pm 0.8$ & $3.1 \pm 0.9$ & $0.019^{\#}$ \\
\hline GAF at admission & $46.1 \pm 10.2$ & $41.2 \pm 12.0$ & n.s. $\#$ \\
\hline GAF at discharge & $64.4 \pm 11.6$ & $67.0 \pm 11.6$ & n.s. $\#$ \\
\hline GAF change & $+18.3 \pm 14.7$ & $+25.8 \pm 14.5$ & $0.026^{\#}$ \\
\hline Duration of the current symptom manifestation, months & $2.6 \pm 0.5$ & $2.1 \pm 0.8$ & $0.011^{\#}$ \\
\hline Inpatient treatment duration, months & $2.0 \pm 1.4$ & $1.3 \pm 1.2$ & $0.056^{*}$ (trend) \\
\hline Duration of the mental disorder, years & $4.9 \pm 6.5$ & $5.1 \pm 8.1$ & n.s. ${ }^{*}$ \\
\hline Number of hospitalizations & $2.1 \pm 3.3$ & $2.6 \pm 4.9$ & n.s.\# \\
\hline Number of somatic diagnoses & $0.7 \pm 0.8$ & $1.1 \pm 1.5$ & $0.070 *$ (trend) \\
\hline School examination level & $3.5 \pm 1.1$ & $3.0 \pm 1.7$ & n.s. ${ }^{\circ}$ \\
\hline Occupational situation (low score $=$ better) & $4.7 \pm 3.8$ & $7.3 \pm 4.0$ & $0.007 / 0.085^{\circ}$ (trend) \\
\hline General psychopharmacological treatment (versus none) & $16 / 2$ & $84 / 11$ & n.s."\# \\
\hline Use of antidepressants (versus none)(trend) & $14 / 4$ & $53 / 42$ & $0.083^{\#}$ \\
\hline Pharmacological problems (versus none) & $1 / 15$ & $11 / 71$ & n.s. \\
\hline Addiction disorder (versus none) & $3 / 15$ & $34 / 61$ & n.s. $\#$ \\
\hline
\end{tabular}

SD, standard deviation, ns, not significant. *t-test; ${ }^{\circ}$ chi-square; ${ }^{\# M a n n-W h i t n e y-U-t e s t . ~}$ 
three variables (see above; CGI part 2, delta GAF, use of antidepressants) which correlated or were directly associated with patient satisfaction within the personality disorder group. Pillai trace was $\mathrm{P}<0.001$, with the dependent variables CGI part $2(\mathrm{P}=0.042)$, delta GAF $(\mathrm{P}=0.048)$ and use of antidepressants $(\mathrm{P}=0.059$; trend $)$.

\section{Discussion}

\section{General findings}

This present exploratory investigation was conducted in a general psychiatric clinic (Stade/Germany) providing the health service for a specific geographic region. We found an overall very good patient satisfaction with the inpatient treatment. Both patient groups, those with and without personality disorders showed a significant improvement of their symptoms, however in patients with personality disorders a slightly lower improvement. Accordingly, the treatment satisfaction was slightly, but significantly reduced in patients suffering from personality disorders in comparison to the remaining patients.

\section{Influences of treatment and socio- demographic variables}

To further explore the reduced satisfaction in patients with personality disorders, we aimed to investigate potential influences of treatment and socio-demographic variables on treatment satisfaction in a subsequent analysis, especially concerning the role of psychopharmacological problems which proved to be a significant influencing parameter on patient satisfaction within the general psychiatric population. ${ }^{3}$ However, in the present investigation focusing on personality disorders we found no increased general psychopharmacological drug treatment or psychopharmacological problems in patients with personality disorders compared to other diagnostic groups.

Interestingly, in the group of personality disorders the use of antidepressants in particular (not the general variable psychopharmacological treatment) was associated with satisfaction as a trend, though this patient group was - even under an increased use of antidepressants - overall less satisfied. It can be assumed that the positive effect of the use of antidepressants on patient satisfaction is not sufficient enough to equalize the limited symptom improvement during the course of the therapy. This goes in line with the current state of research providing not enough evidence for a causal use for the application of antidepressants in patients with personality disorders irrespective of comorbid disorders or target symptoms. ${ }^{17}$ A cochrane review suggests beneficial effects with second-generation antipsy- chotics, mood stabilizers, 18 and dietary supplementation by omega- 3 fatty acids in borderline personality disorder, but no significant influence of any drug on total severity and core features of borderline personality disorder. Two studies showed a significant impact on quality of life in case of risperidone and topiramate. 19,20

The personality disorder patient group showed a younger age, a longer symptom duration of the current symptom manifestation, a lower improvement of symptoms and functioning level during the inpatient treatment, despite of a longer treatment duration and the higher use of antidepressants, less somatic diagnoses as well as a better occupational level and a slightly better job situation. However, within the patients with personality disorders, satisfaction correlated only with the improvement of the symptoms and of the global functioning level.

In contrast, patients with personality disorders and those without did not differ in the following variables: gender, school examination level, duration of the mental disorder, number of hospitalizations, general psychopharmacological treatment (versus none), pharmacological problems, symptom severity at admission, addiction disorders. Thus, these variables cannot be considered responsible for the difference of treatment satisfaction.

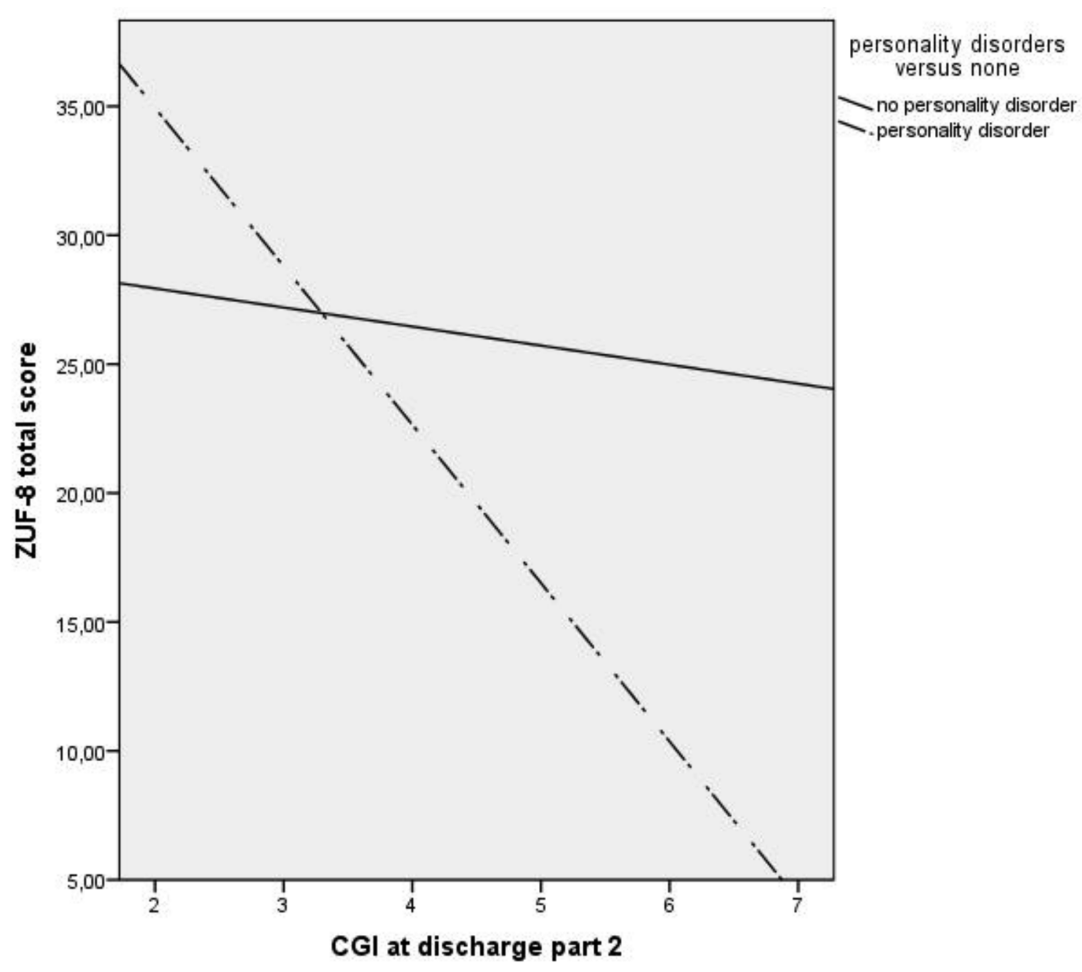

Figure 1. Correlation of treatment satisfaction (ZUF-8 total score) with the CGI score part 2 (low CGI part 2 values = good symptom improvement during the therapy) (patients with personality disorders: $\mathrm{r}=-0.768 ; \mathrm{P}<0.001$; patients without personality disorders: $r=-0.185 ; P=0.072)$.

\section{The role of symptomatology and psychosocial functioning}

Within the group of personality disorders patients clearly improved during the treatment in symptomatology and global functioning $(t$ test for dependent samples). At the time of discharge both the patients with and those without personality disorders showed a sufficient, not significantly differing global functioning level. The multivariate analysis revealed a major influence of the improvement of symptomatology and of the global functioning as well of the use of antidepressants on the patient satisfaction. Thus, the reduced treatment satisfaction in patients with personality disorders seems to be due to less improvement of symptomatology and of global functioning during the treatment (and despite of an increased use of antidepressants); - these both variables seem to have the highest impact, and possibly the role of diagnoses as proposed in previous studies. ${ }^{2}$ Interestingly, treatment satisfaction was much stronger correlated with symptom improvement during the therapy in patients with personality disorders than in patients without personality disorders (Figure 1).

Obviously patients with personality disorders seem to focus especially on the changes of their symptoms during the treatment in order to estimate their satisfaction. It can be sug- 
gested that dysfunctional assumptions which are characteristic in personality disorders might lead to false expectations concerning the treatment success. Additionally, the fact that patients with personality disorders have strong difficulties in personal relationships often affects the therapeutic relationship and can thereby influence the patient satisfaction negatively. A general life dissatisfaction, and therefore also treatment satisfaction, in personality disorders might represent a core feature of the disorder. Investigating the extent to which subjective dissatisfaction is intrinsic to personality disorders, Bouman et al. 10 found the global subjective quality of life to be lower than in patients with (other) major mental disorders, whereas the objective quality of life was mostly significantly poorer among men with major mental disorders than those with personality disorders. A more complex concept of quality of life in patients with personality disorders was supposed compared to patients with major mental disorders for whom almost half of the variance in subjective quality of life rating was related to their everyday activities and their objective sense of safety. ${ }^{10}$

\section{Reference to previous studies}

The reduced satisfaction in personality disorders is a phenomenon which could already be shown in previous studies, $2,21-24$ though its reasons are not understood. Especially the Cluster B personality traits (e.g., antisocial and borderline personality disorder) but also Cluster A as a paranoid personality profile seem to be linked with a decreased treatment satisfaction, in particular with medication treatment, $11,22,25$ whereas dependent personality disorders seem to be less associated with a low treatment satisfaction. ${ }^{21}$ A study of Miller et al. ${ }^{25}$ in 543 psychiatric patients (mostly inpatients) suggests that both maladaptive personality symptoms and general personality traits are predictive of psychiatric treatment utilization and satisfaction. The personality trait openness has been found to be correlated positively with treatment satisfaction. ${ }^{25}$ In that study also a relationship of dissatisfaction with one's medication and the diagnosis of a borderline personality disorder could be detected.

Our investigation can be compared to a survey of treatment satisfaction of 142 psychiatric inpatients in 1993.21 This study of Kelstrup et al. revealed that patients without a personality disorder were more satisfied than patients with antisocial or borderline personality disorders. Patients who received antidepressant medication were much more satisfied with the treatment than patients without antidepressants, whereas there were no significant differences in patients on antipsychotics, benzodiazepines nor individual psychotherapy (versus none, respectively). ${ }^{21}$ The higher satisfaction in patients under antidepressant drugs might be the result of an epiphenomenon of sub-population suffering mostly from an affective disorder, because affective disorders (and also reactive psychoses) have been identified to be associated with more treatment satisfaction than the diagnoses schizophrenia, paranoia as well as transitory adjustment reactions. ${ }^{21}$ We also reported previously of a reduced satisfaction in both personality disorders and schizophrenia. ${ }^{3}$ However, the link of the diagnosis schizophrenia with a reduced treatment satisfaction might be a false positive finding of the hypothesis-testing approach (investigating the group of both personality disorders and schizophrenia together), because it was not mirrored by the current exploratory approach. In some other former studies no difference between psychotic and non-psychotic groups in treatment satisfaction could be found, as well. ${ }^{26,27}$

In 1996 Hueston et al. published a study in 93 patients of a primary care sample. ${ }^{22}$ The authors found lower levels of satisfaction with health care in patients who were at high risk $(n=65)$ for personality disorders compared to those at low risk $(n=28)$. These authors found only a little association between personality disorder type and level of satisfaction. Except for patients with dependent personality tendencies all other personality dimensions showed lower degrees of satisfaction. Furthermore, especially patients at high risk for borderline, schizoid and dependent disorders showed a lower functional status, a higher risk for depression or alcohol abuse in that study, ${ }^{22}$ while medical care utilization was increased in patients with histrionic and dependent disorders. Patients of that study screening positive for narcissistic, schizotypal, antisocial, and obsessive-compulsive disorders were noted to have the least functional impairment, depression, and health care utilization. ${ }^{22}$ These differences could not be explained by demographic or socioeconomic differences. Patients at high risk for personality disorders were more likely to be taking an antidepressant drug. 22

\section{Therapeutic setting}

General psychiatric hospitals are obliged to admit patients with very severe psychiatric disorders, mostly those who have been rejected from specialized psychotherapy clinics for several times because they have not fulfilled their admission criteria (such as therapy motivation). Therefore, one reason for the reduced treatment satisfaction in patients with personality disorders might lie in a lack of a specialized (e.g., psycho-) therapy track, such as - in case of emotional unstable personality disorders - dialectic behavioral therapy, schema therapy, mentalization-based therapy, transference-focused psychotherapy or acceptance and commitment therapy. For example, a combined therapy of fluoxetine with interpersonal psychotherapy adapted for borderline personality disorder proofed to be related to more quality of life as a long-term effect versus single fluoxetine administration. ${ }^{28}$ In case of schema therapy a high treatment satisfaction was demonstrated in 31 patients with severe borderline personality disorder. ${ }^{29}$

According to a recent review the quality of live is seriously impaired in borderline personality disorder patients. ${ }^{30}$ It has to be mentioned that the quality of life concept has similarities to the concept of treatment satisfaction, if quality of life is measured at the end of a therapy; as well, the level of functioning is a determinant of the quality of life. Psychotherapy trials with available empirical data on quality of life (dialectic behavioral therapy, cognitivebehavioral therapy, schema-focused therapy, transference-focused psychotherapy, systems training for emotional predictability and problem solving) show a significant improvement of quality of life, but only the psychotherapy forms dialectic behavioral therapy ${ }^{31}$ and systems training for emotional predictability and problem solving 32 have been shown to be superior to treatment as usual; for cognitive behavioral therapy there was no difference. ${ }^{33,34}$ Furthermore, an inpatient treatment is limited by the conditions of the current form of most acute general psychiatric hospitals (e.g., the comparably short treatment duration) which are still not designed to provide a comprehensive sufficient psychotherapy. Especially for the treatment of personality disorders a personality-oriented and development-oriented understanding of the patient, the developing of an intensive therapeutic relationship and a differentiated macroanalytic diagnostic is necessary in order to initiate therapeutic processes, in particular the transformation of emotional key experiences. ${ }^{35}$ In patients with mood instability such as borderline personality disorders, a patient-centered communication, which acknowledges the patient's experience, may result in a greater patient satisfaction according to a qualitative study. ${ }^{36}$ And treatment satisfaction might appear parallel to dimensions such as personal growth, purpose of life, and changes in positive relations with others. ${ }^{37}$

\section{Limitations}

The study has some limitations. Due to the exploratory nature of the study we refrained from multiple testing. Accordingly, the results have to be rated carefully. Some diagnostic subgroups are very small to consider a valid differentiation in terms of treatment satisfaction. Furthermore, nosological aspects as well as differences in the course of the disease between patients with personality disorders and other psychiatric diagnoses have to be considered. For example, personality disorders 
often appear through the manifestation of another (main) psychiatric diagnosis, such as a depressive syndrome, so that it is difficult to talk of symptom manifestation of the personality disorder. Nevertheless, the comparably heterogeneous sample allows the examination of real world conditions of a typical general psychiatric inpatient population.

\section{Conclusions}

In sum, the reduced satisfaction in patients with personality disorders treated in a general psychiatric hospital seems to be mostly associated with a reduced improvement of global functioning and symptoms. It could be found, that the correlation of symptom improvement and treatment satisfaction is clearly higher in patients with personality disorders compared to patients without, so that symptom improvement has an important impact in inpatients with personality disorders.

Though no empirical data justify the use of psychopharmacological drugs, they are still used in clinical practice. However, our data suggest that patient satisfaction in the subgroup of patients with personality disorders seems to represent a dimension which is much more independent of pharmacological problems than other psychiatric disorders. Probably, psychotherapeutic treatment approaches are much more relevant than psychopharmacological treatments.

In contrast, other variables play a smaller role in personality disorders with respect to treatment satisfaction: the better social status, less somatic symptoms, a longer treatment duration and a higher use of antidepressants and the functioning and symptom level at discharge which were equal to those of the other psychiatric disorders in this study.

The results are of high clinical relevance and are in line with the clinical impression of personality disorders as severe mental diseases requiring intensive care and specialized treatment concepts including changes of public health structures.

\section{References}

1. Min JA, Lee CU, Lee C. Mental health promotion and illness prevention: a challenge for psychiatrists. Psychiatry Investig 2013;10:307-16.

2. Hasler G, Moergeli H, Bachmann R, et al. Patient satisfaction with outpatient psychiatric treatment: the role of diagnosis, pharmacotherapy, and perceived therapeutic change. Can J Psychiatry 2004;49:31521.
3. Gebhardt S, Wolak A, Huber MT. Patient satisfaction and clinical parameters in psychiatric inpatients - the prevailing role of symptom severity and pharmacological disturbances. Compr Psychiatry 2013;54:53-60.

4. Horn K, Martinsen EW. Patient satisfaction after hospitalisation in a psychiatric institution. Tidsskr Nor Laegeforen 2007;127:1506-9.

5. Köhler S, Unger T, Hoffmann S, et al. Patient satisfaction with inpatient psychiatric treatment and its relation to treatment outcome in unipolar depression and schizophrenia. Int J Psychiatry Clin Pract 2015;19:119-23.

6. McHugh RK, Whitton SW, Peckham AD, et al. Patient preference for psychological vs pharmacologic treatment of psychiatric disorders: a meta-analytic review. J Clin Psychiatry 2013;74:595-602.

7. Swoboda E, Kühnel B, Wanders R, König P. Zufriedenheit der Patienten mit der psychiatrischen Versorgung im Krankenhaus. Krankenhauspsychiatrie. 2000;11:13-20.

8. Demyttenaere K, Reines EH, Lönn SL, Lader M. Satisfaction with medication is correlated with outcome but not persistence in patients treated with placebo, escitalopram, or serotonin-norepinephrine reuptake inhibitors: a post hoc analysis. Prim Care Companion CNS Disord 2011;13.

9. Kim JH, Ann JH, Kim MJ. Relationship between improvements of subjective wellbeing and depressive symptoms during acute treatment of schizophrenia with atypical antipsychotics. J Clin Pharm Ther 2011;36:172-8.

10. Bouman YH, Van Nieuwenhuizen C, Schene AH, De Ruiter C. Quality of life of male outpatients with personality disorders or psychotic disorders: a comparison. Crim Behav Ment Health 2008;18:279-91.

11. Dolan M, Millington J. The influence of personality traits such as psychopathy on detained patients using the NHS complaints procedure in forensic settings. Personal Individ Diff 2002;33:955-65.

12. Dilling $\mathrm{H}$, Mombour W. Internationale Klassifikation psychischer Störungen. ICD-10 Kapitel V (F). Bern: Hans Huber, 2013.

13. Schmidt J, Lamprecht F, Wittmann WW. Satisfaction with inpatient management. Development of a questionnaire and initial validity studies. Psychother Psychosom Med Psychol 1989;39:248-55.

14. Cording C, Gaebel W, Spengler A, Stieglitz RD. Die neue psychiatrische Basisdokumentation. Eine Empfehlung der DGPPN zur Qualitätssicherung im (teil)stationären Bereich. Spektrum der Psychiatrie und Nervenheilkunde
1995;24:3-41.

15. Guy W. ECDEU Assessment Manual for Psychopharmacology. Rockville, MD: US Dept. of Health, Education and Welfare, ADAMHA, MIMH Psychopharmacology Research Branch, 1976:218-22.

16. Saß H, Wittchen HU, Zaudig M, Houben I. Diagnostic criteria of the Diagnostic and statistical manual of mental disorders, 4 th ed., revised (DSM-IV-TR). Göttingen: Hogrefe; 2003. pp 47-49.

17. Saunders EF, Silk KR. Personality trait dimensions and the pharmacological treatment of borderline personality disorder. $\mathrm{J}$ Clin Psychopharmacol 2009;29:461-7.

18. Stoffers J, Völlm BA, Rücker G, et al. Pharmacological interventions for borderline personality disorder. Cochrane Database Syst Rev 2010;16:CD005653.

19. Friedel R, Jackson WT, Huston CS, et al. Risperidone treatment of borderline personality disorder assessed by a borderline personality disorder-specific outcome measure: a pilot study. J Clin Psychopharmacol 2008;28:345-7.

20. Loew TH, Nickel MK, Muehlbacher M, et al. Topiramate treatment for women with borderline personality disorder: a doubleblind, placebo-controlled study. J Clin Psychopharmacol 2006;26:61-6.

21. Kelstrup A, Lund K, Lauritsen B, Bech P. Satisfaction with care reported by psychiatric inpatients. Acta Psychiatr Scand 1993;87:374-9.

22. Hueston WJ, Mainous AG 3rd, Schilling R. Patients with personality disorders: functional status, health care utilization, and satisfaction with care. J Fam Pract 1996; 42:54-60.

23. Keith RA. Patient satisfaction and rehabilitation services. Arch Phys Med Rehabil 1998;79:1122-8.

24. Valdes-Stauber J. Patientenzufriedenheit in einer psychiatrischen Institutsambulanz. Ergebnisse einer 8Jahres-Untersuchung. Nervenheilkunde 2010;3:150-6.

25. Miller JD, Pilkonis PA, Mulvey EP. Treatment utilization and satisfaction: examining the contributions of Axis II psychopathology and the Five-Factor Model of personality. J Pers Disord 2006;20:369-87.

26. Hansson L. Patients satisfaction with inhospital psychiatric care. Eur Arch Psychiatry Neurol Sci 1989:239:93-100.

27. Hansson L. The quality of outpatient psychiatric care. Scand J Caring Sci 1989;3: 71-82.

28. Bozzatello P, Bellino S. Combined therapy with interpersonal psychotherapy adapted for borderline personality disorder: a twoyears follow-up. Psychiatry Res 2016;240:151-6.

29. Reiss N, Vogel F, Nill $M$, et al. 
Behandlungszufriedenheit

von

Patientinnen mit Borderline

Persönlichkeitsstörung bei stationärer

Schematherapie. Psychother Psychosom Med Psychol 2013;63:93-100.

30. IsHak WW, Elbau I, Ismail A, et al. Quality of life in borderline personality disorder. Harv Rev Psychiatry 2013;21:138-50.

31. Carter GL, Willcox CH, Lewin TJ, et al. Hunter DBT project: randomized controlled trial of dialectical behaviour therapy in women with borderline personality disorder. Aust N Z J Psychiatry 2010;44: 162-73.

32. Bos EH, van Wel EB, Appelo MT, Verbraak
MJ. A randomized controlled trial of a Dutch version of systems training for emotional predictability and problem solving for borderline personality disorder. J Nerv Ment Dis 2010;198:299-304.

33. Davidson K, Norrie J, Tyrer P, et al.. The effectiveness of cognitive behavior therapy for borderline personality disorder: results from the borderline personality disorder study of cognitive therapy (BOSCOT) trial. J Pers Disord 2006;20: 450-65.

34. Palmer S, Davidson K, Tyrer P, et al. The cost-effectiveness of cognitive behavior therapy for borderline personality disor- der: results from the BOSCOT trial. J Pers Disord 2006;20:466-81.

35. Wendisch M. Verhaltenstherapie emotionaler Schlüsselerfahrungen. Wissenschaftliche Grundlagen und praktische Anleitung. Bern: Hans Huber, 2015.

36. Bilderbeck AC, Saunders KE, Price J, Goodwin GM. Psychiatric assessment of mood instability: qualitative study of patient experience. Br J Psychiatry 2014; 204:234-9.

37. Hasler G, Moergeli H, Schnyder U. Outcome of psychiatric treatment: what is relevant for our patients? Compr Psychiatry 2004;45:199-205. 